\title{
Therapeutic effect of apatinib on overall survival is mediated by prolonged progression-free survival in advanced gastric cancer patients
}

\author{
Lihong Huang ${ }^{1, *}$, Yongyue Wei ${ }^{1, *}$, Sipeng Shen ${ }^{1}$, Qianwen Shi ${ }^{1}$, Jianling Bai ${ }^{1}$, Jin \\ $\mathbf{L i}^{3}$, Shukui Qin ${ }^{4}$, Hao Y $\mathbf{u}^{1}$ and Feng Chen ${ }^{1,2, * *}$ \\ ${ }^{1}$ Department of Biostatistics, School of Public Health, Nanjing Medical University, Nanjing, P.R. China \\ 2 Ministry of Education Key Laboratory for Modern Toxicology, School of Public Health, Nanjing Medical University, Nanjing, \\ P.R. China \\ ${ }^{3}$ Fudan University Shanghai Cancer Center, Shanghai, P.R. China \\ ${ }^{4}$ People's Liberation Army Cancer center, 81st Hospital of People's Liberation Army, Nanjing, Jiangsu, P.R. China \\ * These authors have contributed equally to this work \\ *** Senior author \\ Correspondence to: Hao Yu, email: haoyu@njmu.edu.cn
}

Shukui Qin, email: qinsk@csco.org.cn

Keywords: apatinib, gastric cancer, overall survival, progression-free survival, mediation analysis

Received: September 02, $2016 \quad$ Accepted: October 17,2016 Published: October 25, 2016

Copyright: Huang et al. This is an open-access article distributed under the terms of the Creative Commons Attribution License (CC-BY), which permits unrestricted use, distribution, and reproduction in any medium, provided the original author and source are credited.

\section{ABSTRACT}

Apatinib is reported to significantly improve the overall survival (OS) of patients with advanced gastric cancer who have previously failed second-line chemotherapy. However, it is not well understood whether apatinib acts by improving progression or by prolonging post-progression survival. Here, based on phase III clinical trial data, the mediating effect of apatinib on patient overall survival was systematically quantified, through progression-free survival (PFS), post-progression survival (PPS), and the disease control rate (DCR). PFS was the primary mediator of the association between apatinib treatment and $O S$, with an indirect-effect mean survival time ratio of 1.63 (95\%CI 1.35-1.97), which mediated $93.5 \%$ of the treatment effect. The DCR was also a significant mediator among secondary efficacy endpoints, and had an indirecteffect mean survival time ratio of 1.47 (95\%CI $1.20-1.79,50.9 \%$ mediated). Both primary and other targets of the DCR had similar results. The results indicated that apatinib treatment prolongs progression-free survival rather than post-progression survival, and in turn, leads to improved overall survival. Additionally, our study highlights the value of mediation analysis in clinical trials in providing additional information to build upon traditional primary analysis.

\section{INTRODUCTION}

Though steadily declining in prevalence over the past few decades, gastric cancer remains one of the top three most frequently diagnosed cancers and is the leading cause of cancer-related mortality, both in China and worldwide [1]. Early detection of gastric cancer has a significant impact on survival, as highlighted by the $90 \%$ five-year overall survival (OS) rate of patients whose gastric cancer is detected early. However, when diagnosed at an advanced stage, five-year OS rates range between $10-20 \%[2,3]$. Significant achievements have been demonstrated using first- and second-line chemotherapy for patients with advanced or metastatic gastric cancer [4]; however, after failure of second-line chemotherapy, further treatment options are limited and not widely utilized $[5,6]$.

Recently, we reported that apatinib, a smallmolecule tyrosine kinase inhibitor that selectively inhibits vascular endothelial growth factor receptor 2 , 
Table 1: Baseline demographic and clinical characteristics of full analysis set

\begin{tabular}{|c|c|c|c|}
\hline \multicolumn{2}{|l|}{ Variable } & \multirow{2}{*}{$\begin{array}{l}\text { Apatinib, } n(\%) \\
(N=176) \\
58\end{array}$} & \multirow{2}{*}{\begin{tabular}{|l}
$\begin{array}{l}\text { Placebo, } \boldsymbol{n}(\mathbf{\%}) \\
(\boldsymbol{N = 9 1 )}\end{array}$ \\
58 \\
\end{tabular}} \\
\hline$\Delta$ or varc & Median & & \\
\hline Age, years & Range & $23-71$ & $28-70$ \\
\hline \multirow{2}{*}{ Sex } & Male & $132(75.0)$ & $69(75.8)$ \\
\hline & Female & $44(25.0)$ & $22(24.2)$ \\
\hline \multirow{2}{*}{ ECOG PS } & 0 & $48(27.3)$ & $15(16.5)$ \\
\hline & 1 & $128(72.7)$ & $76(83.5)$ \\
\hline \multirow{2}{*}{ Metastatic sites } & $\leq 2$ & $139(79.0)$ & $71(78.0)$ \\
\hline & $>2$ & $37(21.0)$ & $20(22.0)$ \\
\hline \multirow{2}{*}{ Previous lines of chemotherapy } & 2 & $116(65.9)$ & $58(63.7)$ \\
\hline & $\geq 3$ & $60(34.1)$ & $33(36.3)$ \\
\hline \multirow{3}{*}{ Primary lesion } & Gastric & $69(68.3)$ & $43(71.7)$ \\
\hline & Gastroesophageal junction & $22(21.8)$ & $14(23.3)$ \\
\hline & Unknown & $10(9.9)$ & $3(5.0)$ \\
\hline \multirow{2}{*}{ Prior gastrectomy } & Yes & $122(69.3)$ & $67(73.6)$ \\
\hline & No & $54(30.7)$ & $24(26.4)$ \\
\hline \multirow{4}{*}{ Disease stage } & II & $1(0.6)$ & $1(1.1)$ \\
\hline & III & $10(5.7)$ & $5(5.5)$ \\
\hline & IV & $162(92.0)$ & $83(91.2)$ \\
\hline & Unknown & $3(1.7)$ & $2(2.2)$ \\
\hline \multirow{2}{*}{ Concomitant disease } & Yes & $50(28.4)$ & $31(34.1)$ \\
\hline & No & $126(71.6)$ & $60(65.9)$ \\
\hline
\end{tabular}

significantly improves the prognosis of patients with advanced gastric cancer who have previously failed second-line chemotherapy. Approximately one additional month of progression-free survival (PFS) and two months of prolonged OS were observed [7]. Based on these results, we proposed a new treatment option for patients involving the use of apatinib [8]. It is not clear, however, whether the OS benefit is derived from a prolonged period free of cancer progression, or from post-progression survival (PPS). This information is crucial to the timing of treatment in clinical practice, and recent studies have intensively explored this issue $[9,10]$. However, heterogeneity exists across cancers [11-15].

Causal mediation analysis, a sophisticated epidemiological approach used to determine causal inference, explains the process through which the intervention (in this case, apatinib treatment) affects the outcome (overall survival) through mediators (e.g., progression-free survival) [16-18]. The total effect of the treatment comprises two parts: the indirect effect (the mediating effects) and the direct effect $[19,20]$. Causal pathways from the treatment (exposure) to outcome can be uncovered in prospective epidemiological studies like clinical trials, as shown in a previous study [21].

To clarify the therapeutic benefits of apatinib, we applied mediation analysis to apatinib phase III clinical trial data (ClinicalTrials.gov identifiers NCT01512745). The mediating effect of apatinib on patients' overall survival was systematically quantified through intermediate endpoints including PFS, PPS, and the disease control rate (DCR).
RESULTS

\section{Baseline characteristics}

A total of 273 patients were enrolled in the clinical trial, as described previously [22]. Briefly, five patients in the apatinib group and one in the placebo group withdrew from the study before receiving treatments. Thus, 267 patients were included in the full analysis set (FAS) population, with 176 in the apatinib group and 91 in the placebo group. Baseline characteristics are outlined in Table 1. The demographics and baseline characteristics of the two treatment groups were well balanced.

\section{Prognostic analysis}

Previous reports showed that median OS was significantly improved among patients in the apatinib group compared to the placebo group (6.5 months, $95 \% \mathrm{CI}$ 4.8-7.6 vs. 4.7 months, $95 \%$ CI 3.6-5.4, $p=0.0149$; hazard ratio, $0.709,95 \%$ CI $0.537-0.937, p=0.0156$ ). Similarly, apatinib significantly prolonged the median PFS compared to the placebo (2.6 months, $95 \%$ CI 2.0-2.9 vs. 1.8 months, $95 \%$ CI 1.4-1.9, $p<0.001$; hazard ratio, $0.444,95 \%$ CI $0.331-0.595, p<0.001$ ) [22]. The individual values of PFS and OS results are presented in Figure 1. Most of the PFS values were concentrated below three months for the placebo group and scattered between 0-9 months for the apatinib group. 
Table 2: Mediation analysis of the effect of apatinib treatment on overall survival through intermediate measures after three cycles of treatment

\begin{tabular}{|c|c|c|c|c|c|c|c|c|}
\hline \multirow[b]{2}{*}{ Mediator } & \multicolumn{6}{|c|}{ Full Analysis Set } & \multicolumn{2}{|l|}{ Per Protocol Set } \\
\hline & $\mathbf{N}_{\text {Apatinib }}$ & $\mathbf{N}_{\text {Placebo }}$ & $N D E^{M S T R}(95 \% \mathrm{CI})$ & $\operatorname{NIE}^{M S T R}(95 \% \mathrm{CI})$ & $P$ & \begin{tabular}{|l|} 
Proportion \\
Mediated \\
(\%)
\end{tabular} & NIE $E^{M S T R}(95 \% \mathrm{CI})$ & $P$ \\
\hline PFS & 176 & 91 & $1.03(0.81,1.31)$ & $1.63(1.35,1.97)$ & $<0.001$ & 93.50 & $1.72(1.34,2.32)$ & $<0.001$ \\
\hline PPS $^{\mathbf{a}}$ & 154 & 79 & $1.30(1.11,1.51)$ & $1.11(0.91,1.34)$ & 0.332 & 26.67 & $1.16(0.97,1.43)$ & 0.123 \\
\hline PPS $^{\mathbf{b}}$ & 102 & 70 & $1.26(1.06,1.49)$ & $1.06(0.85,1.28)$ & 0.546 & 21.58 & $1.09(0.91,1.32)$ & 0.406 \\
\hline \multicolumn{9}{|l|}{ DCR } \\
\hline Overall DCR & 176 & 91 & $0.96(0.72,1.28)$ & $1.47(1.20,1.79)$ & $<0.001$ & 50.91 & $1.54(1.25,1.92)$ & $<0.001$ \\
\hline Primary target DCR & 176 & 91 & $1.04(0.78,1.39)$ & $1.23(1.09,1.39)$ & $<0.001$ & 48.91 & $1.30(1.11,1.49)$ & $<0.001$ \\
\hline Other target DCR & 176 & 91 & $1.16(0.87,1.54)$ & $1.09(1.00,1.19)$ & 0.061 & 46.35 & $1.11(1.00,1.22)$ & 0.045 \\
\hline QoL & 176 & 91 & $1.27(0.96,1.69)$ & $1.00(0.96,1.03)$ & 0.816 & 1.64 & $1.02(0.96,1.07)$ & 0.594 \\
\hline
\end{tabular}

PFS: progression-free survival; PPS: post-progression survival; DCR: disease control rate; MSTR: mean survival time ratio. Either NDE or NIE larger than 1.00 represents a favorable survival.

${ }^{\mathrm{a}}$ zero PPS excluded

${ }^{\mathrm{b}}$ censored PFS excluded

\section{Mediation analysis}

Since the patients who received apatinib treatment displayed a longer PFS and OS, we examined whether the effect of apatinib on OS was mainly mediated by the prolonged PFS, or by post-progression activity (Figure 2). Mediation analysis in the FAS population showed that PFS was a major mediator of the treatment effect on patient OS, with an indirect effect MSTR (Mean Survival Time Ratio) of 1.63 (95\% CI 1.35-1.97, $p<0.001)$ that mediated $93.5 \%$ of apatinib's treatment effect (Table 2). Interestingly, after controlling for PFS, the direct effect of apatinib treatment was not significant (Table 2). In addition, after imputing the censored PFS by predicted values of the corresponding AFT model following weibull distribution, the indirect effect MSTR was 1.29 , which retained statistically significant results $(p=0.022)$. On the other hand, PPS was not a significant mediator of treatment effect $(p=0.332)$. This was further supported by sensitivity analysis excluding patients with censored PFS $(p=0.546)$ (Table 2).

We also evaluated two other common short-term prognostic endpoints as potential mediators-DCR (Disease Control Rate) and QoL (Quality of Life) (Figure 2). Patients in the apatinib group received an average of 2.9 cycles of medication, so we assessed DCR and QoL after three cycles of treatment for mediation analysis. About $50.91 \%$ of the apatinib treatment effect on OS was mediated by improving overall DCR $\left(\right.$ NIE $^{\text {MSTR }}=1.47$, $95 \%$ CI $1.20-1.79, p<0.001)$. The primary targets' DCR of patients receiving three cycles of apatinib treatment mediated $48.91 \%$ of the treatment's effect on patients' OS, with statistical significance $\left(N I E^{M S T R}=1.23,95 \%\right.$ CI 1.09-1.39, $p<0.001)$, while a slightly lower indirect effect was identified for other targets' DCR $\left(\right.$ NIE $^{\text {MSTR }}=$ $1.09,95 \%$ CI 1.00-1.19, $p=0.061$ ) (Figure 3B). QoL was not a significant mediator $\left(\right.$ NIE $^{\text {MSTR }}=1.00,95 \%$ CI 0.96 $1.03, p=0.816$ ). Due to small sample size and low ORR (Overall Response Rate), it was not possible to evaluate the mediating effect of ORR.

In addition, we evaluated the mediating effects of DCR when patients received two cycles of treatment. The primary targets' DCR of patients receiving two cycles of apatinib treatment mediated $50.39 \%$ of the treatment effect on patients overall and was statistically significant $\left(N_{I} E^{M S T R}=1.30,95 \%\right.$ CI 1.12-1.47, $\left.p<0.001\right)$, while a slightly lower indirect effect was identified for other targets' DCR $\left(\right.$ NIE ${ }^{M S T R}=1.14,95 \%$ CI $1.03-1.27, p=$ 0.013). Combining these two components, the overall DCR suggested that the indirect-effect mean survival time ratio increased to 1.61 (95\% CI 1.32-1.96) (Figure 3A). However, as mentioned above, after receiving three cycles of treatment, the indirect effects were slightly decreased, as were the mediated proportions, which was likely due to more patients progressing with the disease and ceasing medication.

The sensitivity analysis, which excluded patients with censored OS, exhibited consistent results (Figure 3C, 3D). The mediated proportion (and therefore the indirecteffect mean survival time ratio) was also relatively stable when the covariates were not included in the mediation models (Table 3). Since the consistent results of FAS and PPS increase the confidence of results, sensitivity analysis on per protocol set has been performed accordingly and got similar results (Table 2).

\section{DISCUSSION}

To our knowledge, this is the first quantitative analysis of causal mediation in gastric cancer clinical trials regarding the effects of apatinib treatment on patient progression-free survival, and in turn, overall survival. Our results suggest that the overall survival benefit of 


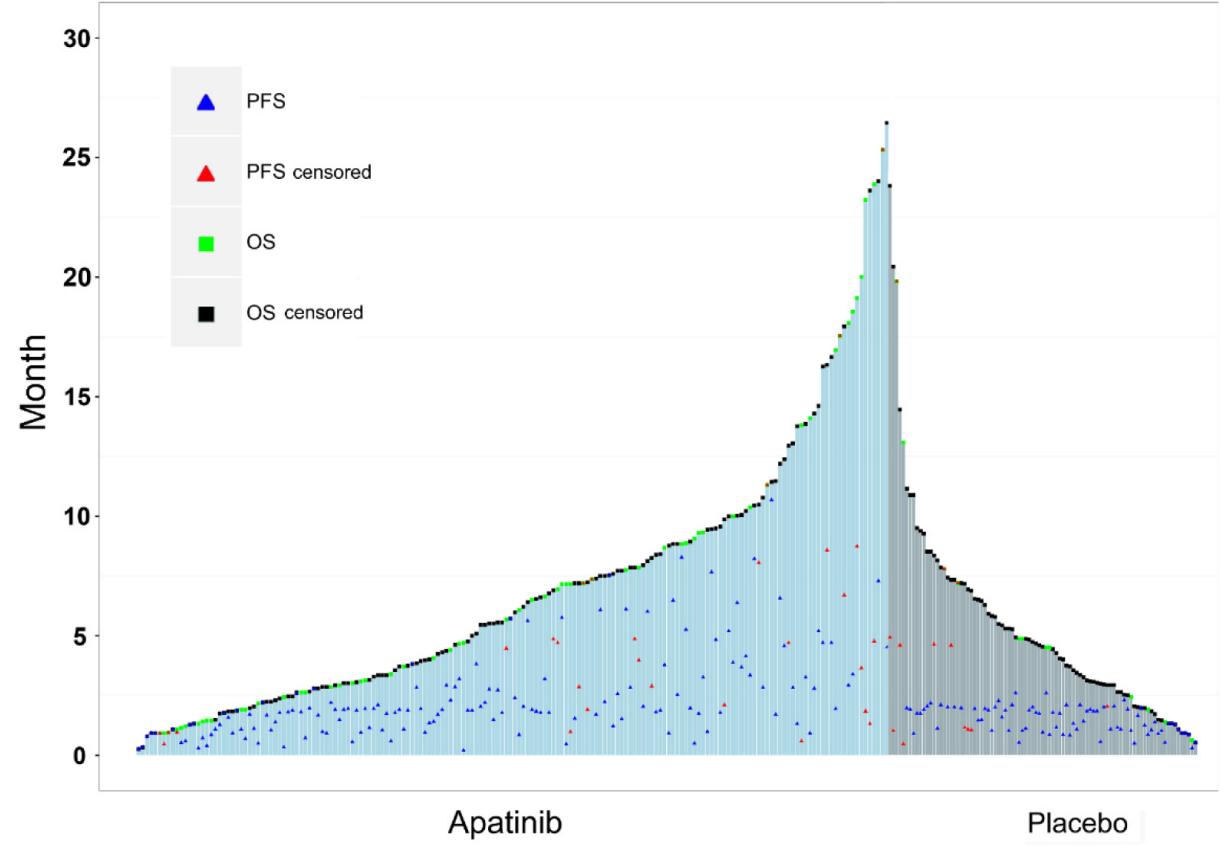

Figure 1: Prognosis of patients receiving apatinib or placebo treatment.

$\longrightarrow$ Indirect effect through the mediator

$\longrightarrow$ Direct effect through pathways beyond the mediator

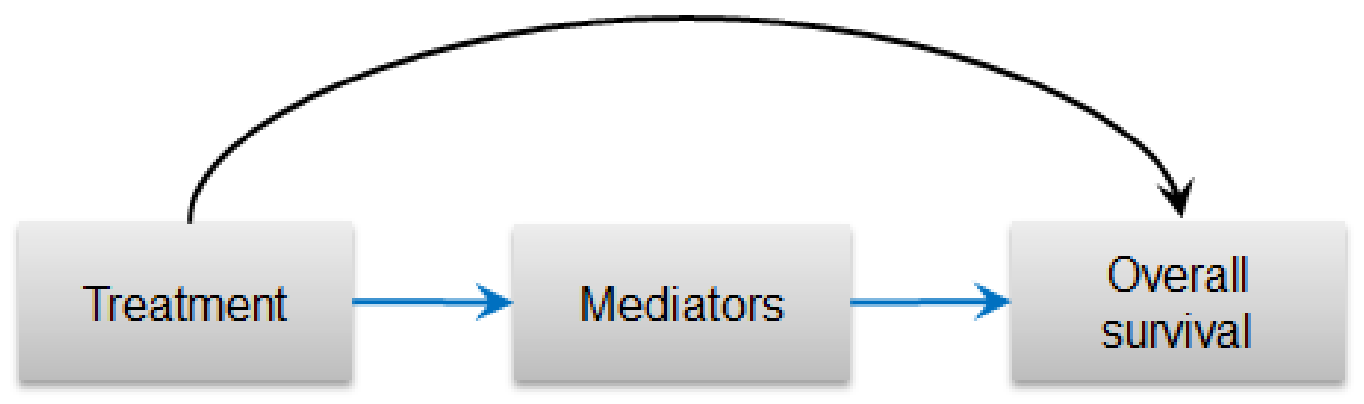

\section{Mediators evaluated}

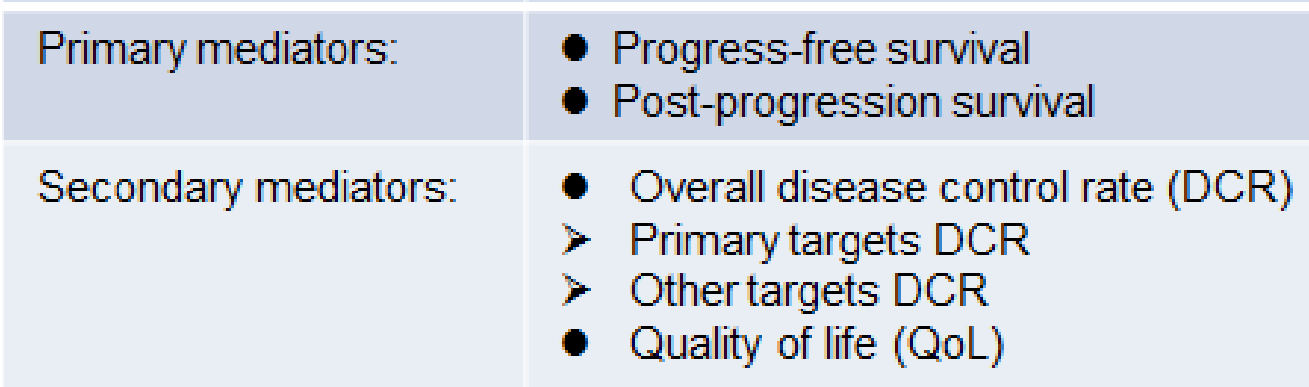

Figure 2: Diagram of mediation analysis. 
Table 3: Sensitivity mediation analysis of with no covariates for the intermediate endpoints after three cycles of treatment

\begin{tabular}{|c|c|c|c|c|c|c|}
\hline \multirow{2}{*}{ Mediator } & \multirow{2}{*}{$\mathbf{N}_{\text {Apatinib }}$} & \multirow{2}{*}{$\mathbf{N}_{\text {Placebo }}$} & \multirow{2}{*}{$\begin{array}{l}N D E^{\mathrm{MSTR}} \\
(95 \% \mathrm{CI})\end{array}$} & \multicolumn{2}{|l|}{ NIE } & \multirow{2}{*}{$\begin{array}{l}\text { Proportion } \\
\text { Mediated(\%) }\end{array}$} \\
\hline & & & & $N I E^{\mathrm{MSTR}}(95 \% \mathrm{CI})$ & $P$ & \\
\hline \multicolumn{7}{|l|}{ Primary endpoint } \\
\hline PFS & 176 & 91 & $1.04(0.79,1.33)$ & $1.61(1.27,2.13)$ & $<0.001$ & 92.66 \\
\hline \multicolumn{7}{|l|}{ Secondary endpoints } \\
\hline Overall DCR & 176 & 91 & $0.99(0.74,1.30)$ & $1.49(1.22,1.82)$ & $<0.001$ & 50.40 \\
\hline Primary targets DCR & 176 & 91 & $1.12(0.85,1.49)$ & $1.20(1.08,1.35)$ & 0.002 & 47.10 \\
\hline Other targets DCR & 176 & 91 & $1.22(0.93,1.61)$ & $1.09(1.00,1.19)$ & 0.056 & 44.91 \\
\hline
\end{tabular}

PFS: progression-free survival DCR: disease control rate; MSTR: median survival time ratio. NDE or NIE larger than 1.00 represents a favorable survival

apatinib treatment was mainly derived from prolonged progression-free survival, rather than post-progression survival. In addition, primary target DCR and other target DCR had similar mediation effects, while overall DCR was slightly higher, indicating that the overall DCR is evaluated by the better response targets.

The primary endpoint should be the variable capable of providing the most clinically relevant and convincing evidence directly related to the primary objective of the trial [23]. In oncology trials, OS is defined as the primary endpoint [9]. Considering that OS requires a longer follow-up time, the correlation between PFS and OS has been extensively studied in various types of cancers to evaluate the validity of PFS as a short-term surrogate. Moriwaki et al. found that the median PFS ratio was correlated with the median OS ratio in advanced biliary tract cancer [11]. Adunlin et al. indicated that PFS can be a suitable surrogate for OS in metastatic breast cancer

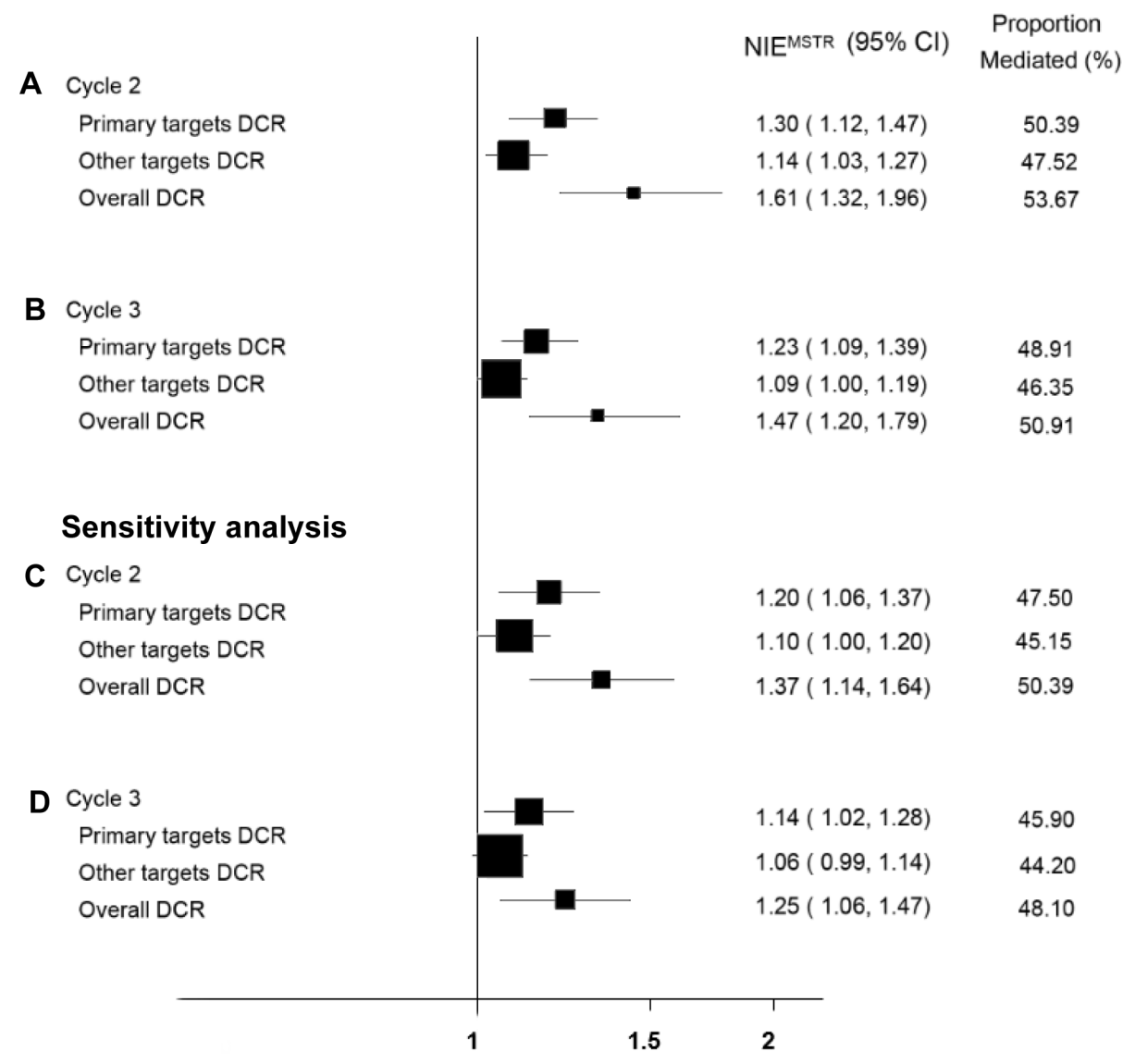

Figure 3: Forest plot for mediation analysis of DCR. Mediation analysis was performed when patients received three A. or two B. cycles of treatment. Sensitivity analysis was performed as well by excluding patients with censored overall survival C., D. 
patients [24]. Thus, PFS may be a reasonable surrogate endpoint for OS. However, in trials of HER2-targeted agents in HER2-positive metastatic breast cancer, PFS moderately correlates with OS at the individual level, suggesting that PFS does not completely substitute for OS in this setting [25]. Kasahara et al. verified that PPS has more impact on OS than PFS in recurrent small cell lung cancer patients [15]. In gastric cancer, Liu et al. reported that PFS is strongly corrected with OS based on a NICE correlation model [26]. Özer-Stillman et al. identified a strong relationship between median OS and PFS among gastrointestinal stromal tumor patients, especially in later lines of therapy based on meta-analysis [27]. However, others reached a different conclusion, questioning the validity of PFS as a surrogate endpoint for OS in gastric cancer patients [28, 29]. Despite this uncertainty, our results highlight the potential and advantage of mediation analysis in evaluating the validity and efficiency of the surrogates. Although the final decision depends on clinical evaluation, our mediation analysis supports the use of surrogates in evaluating the mediation effects beyond statistical correlation.

In addition, mediation analysis quantified the effects of several efficacy variables in clinical trials. For this study, among secondary efficacy endpoints, DCR (overall) was the one and only mediator. QoL was not an effective mediator, indicating that apatinib may not prolong OS by improving the quality of life. The primary endpoint of a confirmatory trial nearly always rests on earlier clinical work carried out in a series of exploratory trials with several different efficacy endpoints [23]. Although the final choice of the primary endpoint depends on clinical evaluation, the mediation analysis results are objective and could be used as supporting analysis in exploratory trials to avoid subjective bias from clinicians.

There are several limitations to our study. First, the validity of our results relies on the assumption that there were no unmeasured confounding variables. To have valid estimates of natural direct and indirect effects, we assumed that there was no unmeasured confounding effect on (1) treatment-OS, (2) mediator-OS, or (3) treatmentmediator, and that there were (4) no mediator-OS confounders effected by treatment [30]. The clinical trial included patients according to the inclusion and exclusion criteria, performed randomization, and was well controlled throughout the trial. Therefore, potential confounders have been controlled. However, the findings were obtained from only one clinical trial; thus, more well studied trials are needed to verify the advantage of the mediation analysis. In addition, the observed strong correlation between PFS and OS in the current study probably be specific for patients have had at least two lines of chemotherapy fail before participating in the study. Thus, the finding should be interpreted with caution among patients receiving firstor second-line treatments.

\section{MATERIALS AND METHODS}

\section{Study population}

The study population was described previously [22]. Briefly, study randomization was stratified according to the number of metastatic sites (more than two sites versus up to two sites). The 273 patients from 32 sites in China, ages 18-70, were histologically confirmed as having advanced or metastatic gastric or gastroesophageal junction adenocarcinoma. Patients were randomized by $2: 1$ to a study group that received apatinib (850 mg once daily) (N $=181)$ or a placebo $(\mathrm{N}=92)$. All participants had at least two lines of chemotherapy fail before participating in the study. Additional enrollment criteria were as follows: at least one measurable lesion as defined by the Response Evaluation Criteria in Solid Tumors (RECIST, version 1.1), Eastern Cooperative Oncology Group (ECOG) performance status of 0 or 1 , and acceptable hematologic, hepatic, and renal function. All patients gave their written informed consent, and approval was obtained from the relevant ethical committees.

There were two primary endpoints: OS and PFS. The time interval before progression or death was PFS. OS was defined as the duration from the time of random assignment to the time of death. Secondary efficacy endpoints included the objective response rate (ORR; including the rate of complete response plus partial response), DCR (including complete response, partial response, and stable disease), and quality of life (QoL; determined using the validated European Organization for Research and Treatment of Cancer Quality of Life Questionnaire Core 30, EORTC QLQ-C30).

A treatment cycle was defined as 28 days (four weeks). Tumor assessments (RECIST) were performed at baseline, after cycles two and three, and every eight weeks thereafter until disease progression was detected. Radiological assessment for disease progression was determined by five independent radiologists from different hospitals. QoL was assessed at baseline, after cycles two and three, and every eight weeks thereafter until disease progression was detected, death occurred, or consent was withdrawn.

\section{Exposure, mediators, outcomes, and covariates}

In this clinical trial, apatinib or placebo treatment was considered to be the exposure variable while the outcome was OS. The PFS was considered as the primary mediator in the mediation analysis (Figure 2), as it was the surrogate endpoint for OS in some oncology clinical trials $[13,31]$. PPS was also evaluated as a potential mediator (Figure 2). To quantify the indirect effects of secondary efficacy endpoints, DCR, and QoL were the analyzed as well (Figure 2). 
We considered age, ECOG status (0 vs. 1), previous chemotherapy (three or more $v s$. two chemotherapy lines), and number of metastatic sites (more than two vs. two or fewer sites) as covariates, in accordance with the multiple Cox model analysis in the original statistical analysis report [7].

\section{Statistical analysis}

There were 3 types of mediators evaluated in this study: continuous (QoL), binary (DCR and ORR), and time-to-event (PFS and PPS). Baseline measurements including age, ECOG status, previous chemotherapy, and number of metastatic sites were considered as covariates (c) in the following models.

\section{Mediation analysis for continuous mediators}

We used the two-step regression method proposed by VanderWeele to estimate the natural direct effect $(N D E)$ and natural indirect effects $(N I E)$ of apatinib $[32,33]$. The mediator model was defined as

$$
M=\beta_{0}+\beta_{1} \cdot \text { treatment }+\beta_{2} \cdot c+e
$$

Assuming OS followed the accelerated failure time (AFT) model, the outcome model was defined as:

$$
\log (\mathrm{OS})=\theta_{0}+\theta_{1} \cdot \text { treatment }+\theta_{2} \cdot M+\theta_{3} c+v \varepsilon
$$

where $c$ represents covariates, and $\varepsilon$ follows the extreme value distribution and $v$ is a shape parameter with $v=1$ if an exponential distribution is assumed or other values if a Weibull distribution is assumed. The $N D E$ and $N I E$ in the scale of mean survival time ratio (MSTR) are defined as:

$$
\begin{aligned}
& N D E^{M S T R}=\exp \left(\theta_{1}\right) \\
& N I E^{M S T R}=\exp \left(\beta_{1} \cdot \theta_{2}\right)
\end{aligned}
$$

\section{Mediation analysis for binary mediator}

The mediator model was re-defined as:

$$
\operatorname{logit}\{P(M=1 \mid A, C)\}=\beta_{0}+\beta_{1} \cdot \text { treatment }+\beta_{2} c
$$

The NDE and NIE were estimated by:

$$
\begin{aligned}
& N D E^{M S T R}=\frac{\exp \left(\theta_{1}\right)\left\{1+\exp \left(\theta_{2}+\beta_{0}+\beta_{2} c\right)\right\}}{\left\{1+\exp \left(\theta_{2}+\beta_{0}+\beta_{2} c\right)\right\}}=\exp \left(\theta_{1}\right) \\
& N I E^{M S T R}=\frac{\left\{1+\exp \left(\beta_{0}+\beta_{2} c\right)\right\}\left\{1+\exp \left(\theta_{2}+\beta_{0}+\beta_{1}+\beta_{2} c\right)\right\}}{\left\{1+\exp \left(\beta_{0}+\beta_{1}+\beta_{2} c\right)\right\}\left\{1+\exp \left(\theta_{2}+\beta_{0}+\beta_{2} c\right)\right\}}
\end{aligned}
$$

\section{Mediation analysis for time-to-event mediator}

Assuming both mediator and outcome (OS) followed the accelerated failure time (AFT) models, the mediator and outcome models were re-defined as:

$$
\begin{aligned}
\log (M) & =\beta_{0}+\beta_{1} \cdot \text { treatment }+\beta_{2} c+v \varepsilon \\
\log (\mathrm{OS}) & =\theta_{0}+\theta_{1} \cdot \text { treatment }+\theta_{2} \cdot \log (M)+\theta_{3} c+v \varepsilon
\end{aligned}
$$

Here, the $N D E^{M S T R}$ and $N I E^{M S T R}$ were estimated by:

$$
\begin{aligned}
& N D E^{M S T R}=\exp \left(\theta_{1}\right) \\
& N I E^{M S T R}=\exp \left(\beta_{1} \cdot \theta_{2}\right)
\end{aligned}
$$

In addition, the proportion of the treatment effect mediated by each variable was calculated by the natural indirect effect divided by the total effect [34]. This metric evaluates the degree to which the treatment's success was influenced by mediators.

In sensitivity analyses, we excluded the predefined covariates to evaluate their impact on the results of the clinical trials. We also restricted the analysis to observations with non-censored OS to better control for confounding by censoring.

Statistical analyses were performed in the full analysis set and conducted using SAS macro software released by Valeri and VanderWeele [35] and $R$ software Version 3.2.3 (The R Foundation for Statistical Computing).

\section{Abbreviations}

DCR, disease control rate

ECOG PS, Eastern Cooperative Oncology Group Performance status

MSTR, mean survival time ratio

$\mathrm{NDE}^{\mathrm{MSTR}}$, mean survival time ratio scale of natural direct-effect

$\mathrm{NIE}^{\mathrm{MSTR}}$, mean survival time ratio scale of natural indirect-effect

ORR, overall response rate

PFS, progression-free survival

PPS, survival post-progression

QoL, quality of life

\section{ACKNOWLEDGMENTS}

We thank Jiangsu Hengrui Medicine, Jiangsu, and Shanghai Hengrui Pharmaceutical, Shanghai, People's Republic of China for providing the clinical trial data.

\section{CONFLICTS OF INTEREST}

None of the authors have any financial or personal conflicts of interest to disclose in relation to this work.

\section{DECLARATIONS}

This study was approved by the institutional review board at each participating center, and conducted in accordance with the International Conference on Harmonization Good Clinical Practice guidelines, the Declaration of Helsinki, and Chinese Law. 


\section{GRANT SUPPORT}

This study was partially supported by the National Natural Science Foundation of China (No. 81402764 to Y Wei, 81302512 to J Bai, 81273184 to H Yu 81473070 and 81530088 to F Chen) and the Natural Science Foundation of Jiangsu, China (No. BK20140907 to Y Wei), Y Wei was also partially supported by A Project Funded by the Priority Academic Program Development of Jiangsu Higher Education Institutions (PAPD) and the Outstanding Young Teachers Training Program of Nanjing Medical University..

\section{REFERENCES}

1. Chen W, Zheng R, Baade PD, Zhang S, Zeng H, Bray F, Jemal A, Yu XQ, He J. Cancer statistics in China, 2015. CA Cancer J Clin. 2016; 66: 115-32. doi: 10.3322/caac.21338.

2. Fock KM, Talley N, Moayyedi P, Hunt R, Azuma T, Sugano K, Xiao SD, Lam SK, Goh KL, Chiba T, Uemura N, Kim JG, Kim N, et al. Asia-Pacific consensus guidelines on gastric cancer prevention. J Gastroenterol Hepatol. 2008; 23: 351-65. doi: 10.1111/j.1440-1746.2008.05314.x.

3. Ono H, Kondo H, Gotoda T, Shirao K, Yamaguchi H, Saito D, Hosokawa K, Shimoda T, Yoshida S. Endoscopic mucosal resection for treatment of early gastric cancer. Gut. 2001; 48: 225-9.

4. (U.S.) NCCN. (2015). NCCN Clinical Practice Guidelines in Oncology: Gastric Cancer (Version 2.2015). https:// www.nccn.org/store/login/login.aspx?ReturnURL=http:// www.nccn.org/professionals/physician_gls/pdf/gastric.pdf.

5. Kang EJ, Im SA, Oh DY, Han SW, Kim JS, Choi IS, Kim JW, Kim YJ, Kim JH, Kim TY, Lee JS, Bang YJ, Lee KW. Irinotecan combined with 5-fluorouracil and leucovorin third-line chemotherapy after failure of fluoropyrimidine, platinum, and taxane in gastric cancer: treatment outcomes and a prognostic model to predict survival. Gastric Cancer. 2013; 16: 581-9. doi: 10.1007/s10120-012-0227-5.

6. Park JS, Lim JY, Park SK, Kim MK, Ko HS, Yoon SO, Kim JW, Choi SH, Cho JY. Prognostic factors of second and third line chemotherapy using 5-fu with platinum, irinotecan, and taxane for advanced gastric cancer. Cancer Res Treat. 2011; 43: 236-43. doi: 10.4143/ crt.2011.43.4.236.

7. Li J, Qin S, Xu J, Xiong J, Wu C, Bai Y, Liu W, Tong J, Liu Y, Xu R, Wang Z, Wang Q, Ouyang X, et al. Randomized, Double-Blind, Placebo-Controlled Phase III Trial of Apatinib in Patients With Chemotherapy-Refractory Advanced or Metastatic Adenocarcinoma of the Stomach or Gastroesophageal Junction. J Clin Oncol. 2016. doi: 10.1200/JCO.2015.63.5995.

8. Brower V. Apatinib in treatment of refractory gastric cancer. Lancet Oncol. 2016. doi: 10.1016/S1470-2045(16)00138-8.

9. Broglio KR, Berry DA. Detecting an overall survival benefit that is derived from progression-free survival. J Natl Cancer
Inst. 2009; 101: 1642-9. doi: 10.1093/jnci/djp369.

10. Morita S, Sakamaki K, Yin G. Detecting overall survival benefit derived from survival postprogression rather than progression-free survival. J Natl Cancer Inst. 2015; 107. doi: 10.1093/jnci/djv133.

11. Moriwaki T, Yamamoto Y, Gosho M, Kobayashi M, Sugaya A, Yamada T, Endo S, Hyodo I. Correlations of survival with progression-free survival, response rate, and disease control rate in advanced biliary tract cancer: a metaanalysis of randomised trials of first-line chemotherapy. $\mathrm{Br}$ J Cancer. 2016; 114: 881-8. doi: 10.1038/bjc.2016.83.

12. Terashima T, Yamashita T, Takata N, Nakagawa H, Toyama T, Arai K, Kitamura K, Sakai Y, Mizukoshi E, Honda M, Kaneko S. Post-progression survival and progression-free survival in patients with advanced hepatocellular carcinoma treated by sorafenib. Hepatol Res. 2015. doi: 10.1111/ hepr.12601.

13. Shitara K, Ikeda J, Yokota T, Takahari D, Ura T, Muro K, Matsuo K. Progression-free survival and time to progression as surrogate markers of overall survival in patients with advanced gastric cancer: analysis of 36 randomized trials. Invest New Drugs. 2012; 30: 1224-31. doi: 10.1007/ s10637-011-9648-y.

14. Michiels S, Pugliano L, Marguet S, Grun D, Barinoff J, Cameron D, Cobleigh M, Di Leo A, Johnston S, Gasparini G, Kaufman B, Marty M, Nekjudova V, et al. Progressionfree survival as surrogate endpoint for overall survival in clinical trials of HER2-targeted agents in HER2-positive metastatic breast cancer. Ann Oncol. 2016. doi: 10.1093/ annonc/mdw132.

15. Kasahara N, Imai H, Kaira K, Mori K, Wakuda K, Ono A, Taira T, Kenmotsu H, Harada H, Naito T, Murakami H, Endo M, Nakajima T, et al. Clinical impact of postprogression survival on overall survival in patients with limited-stage disease small cell lung cancer after first-line chemoradiotherapy. Radiol Oncol. 2015; 49: 409-15. doi: 10.1515/raon-2015-0037.

16. MacKinnon DP, Fairchild AJ, Fritz MS. Mediation analysis. Annu Rev Psychol. 2007; 58: 593-614. doi: 10.1146/ annurev.psych.58.110405.085542.

17. MacKinnon DP. (2008). Introduction to statistical mediation analysis. (New York: Lawrence Erlbaum Associates).

18. Wei Y, Wang Z, Su L, Chen F, Tejera P, Bajwa EK, Wurfel MM, Lin X, Christiani DC. Platelet count mediates the contribution of a genetic variant in LRRC16A to ARDS risk. Chest. 2015; 147: 607-17. doi: 10.1378/chest.14-1246.

19. VanderWeele TJ. Explanation in causal inference : methods for mediation and interaction.

20. Lange T, Hansen JV. Direct and indirect effects in a survival context. Epidemiology. 2011; 22: 575-81. doi: 10.1097/ EDE.0b013e31821c680c.

21. Allison DJ, Ditor DS. Targeting inflammation to influence mood following spinal cord injury: a randomized clinical trial. J Neuroinflammation. 2015; 12: 204. doi: 10.1186/ 
s12974-015-0425-2.

22. Li J, Qin S, Xu J, Xiong J, Wu C, Bai Y, Liu W, Tong J, Liu Y, Xu R, Wang Z, Wang Q, Ouyang X, et al. Randomized, Double-Blind, Placebo-Controlled Phase III Trial of Apatinib in Patients With Chemotherapy-Refractory Advanced or Metastatic Adenocarcinoma of the Stomach or Gastroesophageal Junction. J Clin Oncol. 2016; 34: 144854. doi: 10.1200/JCO.2015.63.5995.

23. ICH Harmonised Tripartite Guideline. Statistical principles for clinical trials. International Conference on Harmonisation E9 Expert Working Group. Stat Med. 1999; 18: 1905-42.

24. Adunlin G, Cyrus JW, Dranitsaris G. Correlation between progression-free survival and overall survival in metastatic breast cancer patients receiving anthracyclines, taxanes, or targeted therapies: a trial-level meta-analysis. Breast Cancer Res Treat. 2015; 154: 591-608. doi: 10.1007/s10549-0153643-5.

25. Michiels S, Pugliano L, Marguet S, Grun D, Barinoff J, Cameron D, Cobleigh M, Di Leo A, Johnston S, Gasparini G, Kaufman B, Marty M, Nekljudova V, et al. Progressionfree survival as surrogate end point for overall survival in clinical trials of HER2-targeted agents in HER2-positive metastatic breast cancer. Ann Oncol. 2016; 27: 1029-34. doi: 10.1093/annonc/mdw132.

26. Liu L, Yu H, Huang L, Shao F, Bai J, Lou D, Chen F. Progression-free survival as a surrogate endpoint for overall survival in patients with third-line or later-line chemotherapy for advanced gastric cancer. Onco Targets Ther. 2015; 8: 921-8. doi: 10.2147/OTT.S82365.

27. Ozer-Stillman I, Strand L, Chang J, Mohamed AF, Tranbarger-Freier KE. Meta-analysis for the association between overall survival and progression-free survival in gastrointestinal stromal tumor. Clin Cancer Res. 2015; 21: 295-302. doi: 10.1158/1078-0432.CCR-14-1779.
28. Paoletti X, Oba K, Bang YJ, Bleiberg H, Boku N, Bouche O, Catalano P, Fuse N, Michiels S, Moehler M, Morita S, Ohashi Y, Ohtsu A, et al. Progression-free survival as a surrogate for overall survival in advanced/recurrent gastric cancer trials: a meta-analysis. J Natl Cancer Inst. 2013; 105: 1667-70. doi: 10.1093/jnci/djt269.

29. Shitara K, Matsuo K, Muro K, Doi T, Ohtsu A. Correlation between overall survival and other endpoints in clinical trials of second-line chemotherapy for patients with advanced gastric cancer. Gastric Cancer. 2014; 17: 362-70. doi: 10.1007/s10120-013-0274-6.

30. Robins JM, Greenland S. Identifiability and exchangeability for direct and indirect effects. Epidemiology. 1992; 3: 14355.

31. Kobayashi Y, Fukui T, Ito S, Shitara K, Hatooka S, Mitsudomi T. Pulmonary metastasectomy for gastric cancer: a 13-year single-institution experience. Surg Today. 2013; 43: 1382-9. doi: 10.1007/s00595-012-0432-1.

32. VanderWeele TJ. Causal mediation analysis with survival data. Epidemiology. 2011; 22: 582-5. doi: 10.1097/ EDE.0b013e31821db37e.

33. Vanderweele TJ. Mediation analysis with multiple versions of the mediator. Epidemiology. 2012; 23: 454-63. doi: 10.1097/EDE.0b013e31824d5fe7.

34. Hafeman DM. "Proportion explained": a causal interpretation for standard measures of indirect effect? Am J Epidemiol. 2009; 170: 1443-8. doi: 10.1093/aje/kwp283.

35. Valeri L, VanderWeele TJ. SAS macro for causal mediation analysis with survival data. Epidemiology. 2015; 26: e23-4. doi: 10.1097/EDE.0000000000000253. 